\title{
Erasure Insertion in Reed-Solomon Coded SFH M-ary FSK with Partial-Band Interference and Rayleigh Fading for Non-Coherent Cooperative Communications
}

\author{
Hoang Anh Ngo, Sohail Ahmed, Lie-Liang Yang and Lajos Hanzo \\ School of Electronics \& Computer Science, University of Southampton, SO17 1BJ, UK \\ Email: \{han08r, sa03r, lly, lh\}@ecs.soton.ac.uk; http://www-mobile.ecs.soton.ac.uk
}

\begin{abstract}
The rationale of our design is that although much of the literature of cooperative systems assumes perfect coherent detection, the assumption of having any channel estimates at the relays imposes an unreasonable burden on the relay station. Hence, non-coherently detected Reed-Solomon (ReS) coded Slow Frequency Hopping (SFH) assisted $M$ ary Frequency Shift Keying (FSK) is proposed for cooperative wireless networks, subjected to both partial-band interference and Rayleigh fading. Erasure insertion (EI) assisted ReS decoding based on the joint maximum output-ratio threshold test (MO-RTT) is investigated in order to evaluate the attainable system performance. Compared to the conventional error-correction-only decoder, the EI scheme may achieve a gain of approximately $3 \mathrm{~dB}$ at the $E_{b} / N_{0}$ of $10^{-4}$, when employing the $\operatorname{Re} S(31,20)$ code combined with 32-FSK modulation. Additionally, we evaluated the system's performance, when either equal gain combining (EGC) or selection combining (SC) techniques are employed at the destination's receiver. The results demonstrated that in the presence of one and two assisting relays, the EGC scheme achieves gains of $1.5 \mathrm{~dB}$ and $1.0 \mathrm{~dB}$ at the $E_{b} / N_{0}$ of $10^{-6}$, respectively, compared to the $\mathbf{S C}$ arrangement.
\end{abstract}

\section{INTRODUCTION}

In order to employ perfect coherent detection, the assumption of having channel estimates at the relays, which is assumed by much of the literature of cooperative systems, imposes an unreasonable burden on the relay station. Hence, in this contribution we investigate lowcomplexity non-coherent detection aided relaying in the absence of channel information. In Slow Frequency Hoping (SFH) assisted $M$ ary Frequency Shift Keying (FSK) employing Reed-Solomon (ReS) coding, typically error-and-erasure decoding is utilized in order to improve the attainable system performance, when subjected to interference, jamming and fading [1], [2]. Several erasure insertion (EI) techniques assisting error-and-erasure $\mathrm{ReS}$ decoding were proposed in [1], [3], namely the ratio-threshold test (RTT), the output threshold test (OTT), and the joint maximum output and ratio threshold test (MO-RTT).

Owing to the ability of improving the achievable performance, the family of Multiple-Input-Multiple-Output (MIMO) techniques [4], [5] has been adopted for various wireless standards. However, it is ineffective to employ multiple antennas in mobile handsets, since their diversity gain remains limited by the spatial correlation imposed by their compact physical dimensions. In order to overcome this obstacle, relaying techniques were proposed, where single-antenna aided mobile stations collaborate to create a distributed MIMO system.

In this contribution, we propose a non-coherently detected ReS coded SFH M-FSK system, subjected to partial-band interference and Rayleigh fading, in the context of wireless cooperative networks. The EI technique will be invoked for error-and-erasure ReS decoding based on the joint MO-RTT technique, along with the detailed

The research leading to these results has received funding from the European Union's Seventh Framework Programme ([FP7/2007-2013]) under grant agreement no [214625]. The financial support of RC-UK under the auspices of the UK-India Advanced Technology Centre as well as that of the China-UK Science Bridge in 4G Wireless Communications is also gratefully acknowledged. analysis of the related decision statistics. With the aid of these decision statistics, the analytical expressions of the $\mathrm{ReS}$ codeword error probability as well as the bit error probability (BEP) achieved by erasure decoding will be derived. Our results show that in the context of the wireless relaying network, the EI ReS decoding scheme may significantly enhance the overall achievable system performance.

The outline of this paper is as follows. Section II describes the system's structure and the associated assumptions. The MO-RTT EI scheme and the related decision statistics are considered in Section III, followed by our numerical results in Section IV. Finally, our concluding remarks are offered in Section V.

\section{SySTEM DESCRIPTION}

The system under consideration consists of a source station (SS), $L$ relay stations (RS) and a destination station (DS) communicating over Rayleigh fading channels subjected to Partial-Band Gaussian Interference (PBGI). The communication process is divided into two time slots. In the first slot, the SS broadcasts its packets to all the RSs and to the DS. In the second slot, each RS will forward the re-encoded packets to the DS, if it correctly decoded the received information. Otherwise, the packet will be ignored at the RS.

At the SS, the data bits are first encoded by the $(N, K) \operatorname{ReS}$ code defined over the Galois field $G F\left(2^{b}\right)$, which turns $K b$-bit uncoded symbols into $N$ ReS-encoded symbols. Subsequently, the encoded symbols are passed to the $M$-ary FSK modulator. We assume that $M=N=2^{b} \geq 4$, so that each $b$-bit ReS coded symbol describes an $M$-ary FSK symbol. Finally, the frequency synthesizer, which operates under the control of a pseudo noise (PN) generator, generates a sequence of random hopping frequencies, one of which is activated during each hop interval of duration $T_{h}$, or symbol interval $T_{s}$, where we assume $T_{h}=T_{s}$. To allow non-coherent detection at the receiver, the bandwidth of a single frequency hopping $(\mathrm{FH})$ tone is given by $B=1 / T_{h}$. The transmit signal at the SS may be modelled as

$$
x_{i}(t)=\sqrt{2 E_{s} R_{c}} \cos \left\{2 \pi\left(f_{n}+f_{i}\right) t+\varphi_{n}+\varphi_{i}\right\},
$$

where $E_{s}$ is the symbol power at the SS, $R_{c}=K / N$ is the ReS code-rate; $f_{n}$ is the hopping frequency during the $n^{t h}$ FH interval and $f_{i}$ is the $i^{t h}$ frequency associated with the $i^{t h}$ transmitted MFSK/ReS symbol. Finally, $\varphi_{n}$ and $\varphi_{i}$ are random phases during the $n^{\text {th }} \mathrm{FH}$ interval and the $i^{\text {th }}$ symbol interval.

The modulated signal of each FH tone is transmitted over the frequency-flat fading channels obeying the Rayleigh distribution having the probability density function (p.d.f) given by

$$
f(|h|)=\frac{2|h|}{\Omega} e^{-|h|^{2} / \Omega},
$$

where we have $\Omega=E\left[|h|^{2}\right]$.

Furthermore, the communication channels are assumed to be contaminated by both PBGI and additive white Gaussian noise (AWGN). The PBGI occupies a fraction of $\rho \leq 1$ band, having the power spectral density of $N_{I} / 2 \rho$, while the AWGN has the power spectral 
density of $N_{0} / 2$. Consequently, in the specific portion of the band interfered by the PBGI, the total noise power spectral density is $N_{n} / 2=N_{0} / 2+N_{I} / 2$, while it is $N_{n} / 2=N_{0} / 2$ in the remainder of the band.

As a result, the signal received at the $l^{\text {th }}$ RS may be expressed as $r_{i l}(t)=G_{s r_{l}} h_{i, s r_{l}} \sqrt{2 E_{s} R_{c}} \cos \left\{2 \pi\left(f_{n}+f_{i}\right) t+\phi_{i, s r_{l}}\right\}+n_{i, s r_{l}}(t)$,

where $G_{s r}$ is the pathloss-reduction related gain [6], $h_{i, s r_{l}}$ is the fading coefficient of the $l^{\text {th }}$ Source-to-Relay (SR) link, $n_{i, s r_{l}}$ is the effective noise as defined above and $\phi_{i, s r_{l}}$ includes all the phases in the received signal due to frequency hopping, carrier- and MFSKmodulation as well as that induced by the fading.

After demodulation, the RS will demodulate and ReS-decode the packet. If the packet is correctly ReS-decoded, it will be re-encoded, re-modulated and forwarded to the DS. Otherwise, the RS will ignore the packet and turn to idle mode, waiting for a new packet to arrive from the SS. The probability of a packet being forwarded from the RS to the DS will be detailed in Section III.

Finally, the signal received at the DS may be expressed as

$$
y_{i}(t)=\sum_{l=0}^{L} G_{l} h_{i, l} \sqrt{2 E_{l} R_{c}} \cos \left\{2 \pi\left(f_{n}+f_{i}\right) t+\phi_{i, l}\right\}+n_{i, l}(t),
$$

where $G_{l}$ is the pathloss-reduction related gain of the $l^{\text {th }}$ Relay-toDestination (RD) link, while $h_{i, l}$ is the fading coefficient of the $l^{\text {th }}$ RD link. In Eq. (4), the direct Source-to-Destination (SD) link is represented by $l=0$, where we have $h_{i, 0}=h_{i, s d}, G_{i, 0}=G_{i, s d}$ and $n_{i, 0}=n_{i, s d}$. Again, it is assumed that the fading and noise variances of the RD links are identical. Meanwhile, $E_{l}$ represents the transmit power at the relay. In order to make the comparison between the equal gain combining technique and the selection combining technique at the destination's receiver convenient, $E_{l}$ is set to $E_{s} \frac{G_{l}}{G_{0}}$.

The ReS channel decoders employed at both the RS and the DS rely error-and-erasure decoding in order to improve the achievable performance. In this treatise, our analysis will be focused on the MO-RTT technique, which outperforms both the OTT and the RTT techniques [1], [2].

\section{RES-CODED SYSTEM USING ERROR-AND-ERASURE DECODING}

\section{A. At the Relay}

The transmissions from the SS to the relays may be viewed as traditional direct communications between two nodes. Thus, according to [7], the codeword decoding error probability after "errors-anderasures" $\operatorname{ReS}(N, K)$ decoding at the $l^{\text {th }}$ relay can be expressed as:

$$
P_{w}=\sum_{i=0}^{N} \sum_{j=j_{0}(i)}^{N-i}\left(\begin{array}{c}
N \\
i
\end{array}\right)\left(\begin{array}{c}
N-i \\
j
\end{array}\right) P_{t, l}^{i} P_{e, l}^{j}\left(1-P_{t, l}-P_{e, l}\right)^{N-i-j}
$$

where $j_{0}(i)=\max \{0, N-K+1-2 i\}$, while $P_{e, l}$ and $P_{t, l}$ represent the symbol erasure probability and random symbol error probability before ReS decoding, which are given by Eqs. (38) and (39) of [1] for the MO-RTT.

Hence, the probability of the $l^{t h}$ relay actively forwarding packets to the DS is given by

$$
P_{R f}=1-P_{w} .
$$

\section{B. At the Destination}

1) Symbol Error Probability (SEP): The choice of the diversity combining technique at the destination's receiver significantly affects the achievable SEP performance. For non-coherent detection systems, the EGC and SC are frequently employed. Hence, we will investigate the SEP of these two diversity combining techniques here.

$\boldsymbol{E G C}$ : Based on the assumptions of Section II, the outputs of the low complexity non-coherent square-law detector dispensing with any channel estimates at the DS's receiver may be expressed as

$$
\begin{gathered}
U_{1}=\sum_{l=0}^{L_{f}}\left|G_{l} h_{l} \sqrt{P_{l} R_{c}} e^{-j \phi_{l}}+n_{1, l}\right|^{2}, \\
U_{i}=\sum_{l=0}^{L_{f}}\left|n_{i, l}\right|^{2}, \quad i=2,3, \ldots, M,
\end{gathered}
$$

where $L_{f}$ is the number of relays forwarding packets to the DS.

According to [8], the p.d.fs of the outputs $U_{1}$ and $U_{i}$ are given as

$$
\begin{aligned}
& f_{U_{1}}(y)=\frac{y^{L_{f}}}{\left(1+\overline{\gamma_{l}}\right)^{L_{f}+1} \cdot L_{f} !} \exp \left(-\frac{y}{1+\overline{\gamma_{l}}}\right), \quad y \geq 0, \\
& f_{U_{i}}(y)=\frac{y^{L_{f}}}{L_{f} !} \exp (-y), \quad i=2,3 \ldots M ; \quad y \geq 0,
\end{aligned}
$$

where we have $\overline{\gamma_{l}}=|\bar{h}|^{2} P_{s} / N_{n}$.

When there are $L_{f}$ relays forwarding packets to the DS, the average erroneous symbol probability of $P_{N_{n}, L_{f}}\left(H_{0}\right)$, where $H_{0}$ presents the hypotheses of erroneous decision, at the DS can be expressed as [9]

$$
\begin{aligned}
P_{N_{n}, L_{f}}\left(H_{0}\right)= & 1-\int_{0}^{\infty} f_{U_{1}}(y)\left[\int_{0}^{y} f_{U_{2}}(x) d x\right]^{M-1} d y \\
= & \frac{1}{L_{f} !} \sum_{m=1}^{M-1} \frac{(-1)^{M-1}\left(\begin{array}{c}
M-1 \\
m
\end{array}\right)}{\left(1+m+m \overline{\gamma_{l}}\right)^{L_{f}+1}} \\
& \cdot \sum_{k=0}^{m L_{f}} \beta_{k m}\left(L_{f}+k\right) !\left(\frac{1+\overline{\gamma_{l}}}{1+m+m \overline{\gamma_{l}}}\right)^{k}
\end{aligned}
$$

where $\beta_{k m}$ is the set of coefficients satisfying the following condition

$$
\left(\sum_{k=0}^{L_{f}} \frac{U_{1}^{k}}{k !}\right)^{k}=\sum_{k=0}^{m L_{f}} \beta_{k m} U_{1}^{k}
$$

$S C$ : The outputs of the low complexity non-coherent square-law detector requiring no channel estimates at the DS's receiver may be expressed as

$$
\begin{aligned}
& U_{1}=\max \left\{U_{1, l}\right\}, \quad l=0,1, . ., L_{f}, \\
& U_{i}=\max \left\{U_{i, l}\right\}, \quad i=2,3, \ldots, M,
\end{aligned}
$$

where $U_{1, l}=\left|G_{l} h_{l} \sqrt{P_{l} R_{c}} e^{-j \phi_{l}}+n_{1, l}\right|^{2}$ and $U_{i, l}=\left|n_{i, l}\right|^{2}$.

According to [10], the p.d.fs of the outputs $U_{1}$ and $U_{i}$ are given as

$f_{U_{1}}(y)=\frac{L_{f}+1}{1+\gamma_{l}} \exp \left(-\frac{y}{1+\gamma_{l}}\right)\left[1-\exp \left(-\frac{y}{1+\gamma_{l}}\right)\right]^{L_{f}}, y \geq 0$

$f_{U_{i}}(y)=\left(L_{f}+1\right) \exp (-y)[1-\exp (-y)]^{L_{f}}, \quad i=2,3 \ldots M ; y \geq 0$.

It also transpired from [10] that when there are $L_{f}$ relays forwarding error-free packets to the DS, the average erroneous symbol probability of $P_{N_{n}, L_{f}}\left(H_{0}\right)$ at the DS can be expressed as

$$
\begin{aligned}
P_{N_{n}, L_{f}}\left(H_{0}\right)= & 1-\sum_{l=1}^{L_{f}+1}(-1)^{l+1}\left(\begin{array}{c}
L_{f}+1 \\
l
\end{array}\right) \\
& \cdot \prod_{m=1}^{\left(L_{f}+1\right)(M-1)} \frac{m}{m+l /\left(1+\gamma_{c}\right)} .
\end{aligned}
$$


SEP: The average erroneous symbol probability of $P_{N_{n}}\left(H_{0}\right)$ at the DS is given by

$$
P_{N_{n}}\left(H_{0}\right)=\sum_{L_{f}=0}^{L}\left(\begin{array}{c}
L \\
L_{f}
\end{array}\right) P_{R f}^{L_{c}}\left(1-P_{R f}\right)^{L-L_{c}} \cdot P_{N_{n}, L_{f}}\left(H_{0}\right),
$$

while the correct symbol probability is

$$
P_{N_{n}}\left(H_{1}\right)=1-P_{N_{n}}\left(H_{0}\right) \text {. }
$$

where $H_{1}$ presents the hypotheses of correct decision

Since the fraction $\rho$ of the band is interfered with, the average erroneous and correct symbol probabilities may be expressed as

$$
\begin{aligned}
& P\left(H_{0}\right)=(1-\rho) P_{N_{0}}\left(H_{0}\right)+\rho P_{N_{0}+N_{i} / \rho}\left(H_{0}\right), \\
& P\left(H_{1}\right)=1-P\left(H_{0}\right) .
\end{aligned}
$$

2) Statistics of the Erasure Insertion Related Variables: Let $\left\{U_{1, l}, U_{2, l}, \ldots, U_{M, l}\right\}$ represent the decision variables input to the MFSK demodulator. We denote the maximum and the "second" maximum of $\left\{U_{1, l}, U_{2, l}, \ldots, U_{M, l}\right\}$ by

$$
\begin{aligned}
& Y_{1}=\max _{1}\left\{U_{1, l}, U_{2, l}, \ldots, U_{M, l}\right\}, \\
& Y_{2}=\max _{2}\left\{U_{1, l}, U_{2, l}, \ldots, U_{M, l}\right\},
\end{aligned}
$$

and the ratio of the "second" maximum to the maximum is formulated as

$$
\lambda=\frac{Y_{2}}{Y_{1}}, \quad 0 \leq \lambda \leq 1
$$

In the context of the joint MO-RTT, the erasure insertion is based on the observation of both the maximum $Y_{1}$ of (22) and the ratio $\lambda$ of (24). Therefore, the joint p.d.fs of $f_{Y_{1}, \lambda}\left(y, r \mid H_{1}\right)$ and $f_{Y_{1}, \lambda}\left(y, r \mid H_{0}\right)$ have to be derived, in order to evaluate the error-and-erasure $\operatorname{ReS}$ decoding performance in term of the joint MO-RTT erasure insertion scheme.

According to [1], the joint conditional p.d.fs $f_{Y_{1}, \lambda}\left(y, r \mid H_{1}\right)$ and $f_{Y_{1}, \lambda}\left(y, r \mid H_{0}\right)$ associated with the MO-RTT based erasure insertion scheme may be expressed as

$$
\begin{aligned}
& f_{Y_{1}, \lambda}\left(y, r \mid H_{1}\right)= \\
& \quad \frac{(M-1) y}{P_{N_{n}}\left(H_{1}\right)} f_{U_{1}}(y) f_{U_{m}}(y r)\left[\int_{0}^{y r} f_{U_{j}}(x) d x\right]^{M-2}, \\
& 0 \leq y<\infty, 0 \leq r \leq 1 \\
& f_{Y_{1}, \lambda}\left(y, r \mid H_{0}\right)= \\
& \quad \frac{(M-1) y}{P_{N_{n}}\left(H_{0}\right)}\left\{f_{U_{m}}(y) f_{U_{1}}(y r)\left[\int_{0}^{y r} f_{U_{j}}(x) d x\right]^{M-2}\right. \\
& \quad+(M-2) f_{U_{m}}(y) f_{U_{j}}(y r)\left[\int_{0}^{y r} f_{U_{1}}(x) d x\right] \\
& \left.\quad\left[\int_{0}^{y r} f_{U_{k}}(x) d x\right]^{M-3}\right\}, 0 \leq y<\infty, 0 \leq r \leq 1,
\end{aligned}
$$

where $f_{U_{1}}(y)$ represents the p.d.f of (9) and (15), while, $f_{U_{m}}(y)$, $f_{U_{j}}(y)$ and $f_{U_{k}}(y)$ represent the p.d.f of (10) and (16).

EGC: Substituting (9) and (10) into Eqs. (25) and (26) and employing the function (2.321.2) of [11], the joint p.d.fs of $Y_{1}$ and $\lambda=Y_{2} / Y_{1}$ under the hypotheses $H_{1}$ of correct decision and $H_{0}$ of erroneous decision, respectively, can be expressed as

$$
\begin{aligned}
f_{Y_{1}, \lambda}\left(y, r \mid H_{1}\right)= & \frac{(M-1) y}{P_{N_{n}}\left(H_{1}\right)} \\
& \cdot \frac{y^{2 L_{f}} r^{L_{f}}}{\left(1+\bar{\gamma}_{l}\right)^{L_{f}+1} \cdot\left(L_{f} !\right)^{M}} e^{\left(-\frac{1+r+r \overline{\gamma_{l}}}{1+\bar{\gamma}_{l}} y\right)} \\
& \cdot\left[L_{f} !-e^{-y r} \sum_{k=0}^{L_{f}} k !\left(\begin{array}{c}
L_{f} \\
k
\end{array}\right)(y r)^{L_{f}-k}\right]^{M-2},
\end{aligned}
$$

$$
\begin{aligned}
f_{Y_{1}, \lambda}\left(y, r \mid H_{0}\right)= & \frac{(M-1) y}{P_{N_{n}}\left(H_{0}\right)} \cdot \frac{y^{2 L_{f}} r^{L_{f}}}{\left(1+\bar{\gamma}_{l}\right)^{L_{f}+1} \cdot\left(L_{f} !\right)^{M}} \\
& \left\{e ^ { ( - \frac { 1 + r + r \overline { \gamma } _ { l } } { 1 + \overline { \gamma } _ { l } } y ) } \left[L_{f} !-e^{-y r} \sum_{k=0}^{L_{f}} k !\left(\begin{array}{c}
L_{f} \\
k
\end{array}\right)\right.\right. \\
& \left.\cdot(y r)^{L_{f}-k}\right]^{M-2}+(M-2) e^{-(r+1) y} \\
& \cdot\left[\left(1+\overline{\gamma_{l}}\right)^{L_{f}+1} L_{f} !-e^{\left(-\frac{y r}{1+\bar{\gamma}_{l}}\right)}\right. \\
& \left.\cdot \sum_{k=0}^{L_{f}}\left(1+\overline{\gamma_{l}}\right)^{k+1} k !\left(\begin{array}{c}
L_{f} \\
k
\end{array}\right)(y r)^{L_{f}-k}\right] \\
& \left.\cdot\left[L_{f} !-e^{-y r} \sum_{k=0}^{L_{f}} k !\left(\begin{array}{c}
L_{f} \\
k
\end{array}\right)(y r)^{L_{f}-k}\right]^{M-3}\right\} .
\end{aligned}
$$

SC: Similarly, substituting (15) and (16) into Eqs. (25) and (26), we have

$$
\begin{aligned}
f_{Y_{1}, \lambda}\left(y, r \mid H_{1}\right)= & \frac{(M-1) y}{P_{N_{n}}\left(H_{1}\right)} \cdot \frac{\left(L_{f}+1\right)^{2}}{1+\gamma_{l}} e^{\left(-\frac{1+r+r \gamma_{l}}{1+\gamma_{l}} y\right)} \\
f_{Y_{1}, \lambda}\left(y, r \mid H_{0}\right)= & \frac{(M-1) y}{P_{N_{n}}\left(H_{1}\right)} \cdot\left\{\frac{\left(L_{f}+1\right)^{2}}{1+\gamma_{l}} e^{\left.\left(-\frac{y}{1+\gamma_{l}}\right)\right]^{L_{f}}\left[1-e^{-y r}\right]^{\left(L_{f}+1\right)(M-2)+L_{f}}},\right. \\
& \cdot\left[1-e^{\left(-\frac{y}{1+\gamma_{l}}\right)}\right]^{L_{f}}\left[1-e^{-y r}\right]^{\left(L_{f}+1\right)(M-2)+L_{f}} \\
& +(M-2) \cdot \frac{\left(L_{f}+1\right)^{3}}{2} e^{(-y r-y)}\left[1-e^{-y}\right]^{L_{f}} \\
& \left.\cdot\left[1-e^{\left(-\frac{y r}{1+\gamma_{l}}\right)}\right]^{2}\left[1-e^{-y r}\right]^{\left(L_{f}+1\right)(M-3)+L_{f}}\right\} .
\end{aligned}
$$

3) Codeword Error Probability: Based on the statistics formulated above, the symbol erasure probability, $P_{e}$, and the random symbol error probability, $P_{t}$, corresponding to the noise power spectral of $N_{n}$, and the given thresholds of $Y_{T}$ and $\lambda_{T}$, may be expressed as [1]

$$
\begin{aligned}
P_{e}\left(N_{n}, Y_{T}, \lambda_{T}\right)= & P_{N_{n}}\left(H_{1}\right) \int_{0}^{Y_{T}} \int_{\lambda_{T}}^{1} f_{Y_{1}, \lambda}\left(y, r \mid H_{1}\right) d r d y \\
& +P_{N_{n}}\left(H_{0}\right) \int_{0}^{Y_{T}} \int_{\lambda_{T}}^{1} f_{Y_{1}, \lambda}\left(y, r \mid H_{0}\right) d r d y,(31) \\
P_{t}\left(N_{n}, Y_{T}, \lambda_{T}\right)= & P_{N_{n}}\left(H_{0}\right)\left[1-\int_{0}^{Y_{T}} \int_{\lambda_{T}}^{1} f_{Y_{1}, \lambda}\left(y, r \mid H_{0}\right) d r d y\right] .
\end{aligned}
$$

Due to the effect of the PBGI, the symbol erasure probability and the random symbol error probability after erasure filling based decoding at the destination may be presented as [1]

$$
\begin{gathered}
P_{e}=(1-\rho) P_{e}\left(N_{0}, Y_{T}, \lambda_{T}\right)+\rho P_{e}\left(N_{0}+N_{I} / \rho, Y_{T}, \lambda_{T}\right), \\
P_{t}=(1-\rho) P_{t}\left(N_{0}, Y_{T}, \lambda_{T}\right)+\rho P_{t}\left(N_{0}+N_{I} / \rho, Y_{T}, \lambda_{T}\right) .
\end{gathered}
$$

As a result, the codeword error probability $P_{w}$ at the destination may be expressed as

$$
P_{w}=\sum_{i=0}^{N} \sum_{j=j_{0}(i)}^{N-i}\left(\begin{array}{c}
N \\
i
\end{array}\right)\left(\begin{array}{c}
N-i \\
j
\end{array}\right) P_{t}^{i} P_{e}^{j}\left(1-P_{t}-P_{e}\right)^{N-i-j},
$$

where $j_{0}(i)=\max \{0, N-K+1-2 i\}$. 
Consequently, the symbol error probability $P_{s}$ after error-anderasure $\mathrm{ReS}$ decoding may be expressed as

$P_{s}=\frac{1}{N} \sum_{i=0}^{N} \sum_{j=j_{0}(i)}^{N-i}(i+j)\left(\begin{array}{c}N \\ i\end{array}\right)\left(\begin{array}{c}N-i \\ j\end{array}\right) P_{t}^{i} P_{e}^{j}\left(1-P_{t}-P_{e}\right)^{N-i-j}$,

while the bit error probability $P_{b}$ may be approximated as [9]

$$
P_{b}=\frac{2^{b-1}}{M-1} P_{s} .
$$

\section{Performance Results}

Based on the expressions formulated in the previous sections, we will investigate the performance of the low-complexity non-coherent FFH MFSK ReS coded systems operating in a cooperative network. For all the performance results below, the channels are assumed to be flat Rayleigh faded. The signal power to interference plus noise ratio (SINR) is set at $10 \mathrm{~dB}$, while the portion of the band, which is interfered by the PBGI, equals 0.15 . All relays are assumed to be at the mid-point of the source-to-destination link.

Fig. 1 portrays the relay's codeword error probability at $E_{b} / N_{0}=$ $8 d B$ when utilizing the joint MO-RTT decoding technique. The $\operatorname{Re} S(31,20)$ code and 32-ary FSK modulation are employed. According to the figure, there exists an optimum threshold value of $Y_{T}$ or $\lambda_{T}$, which minimizes the codeword error probability. It is noted that when we have $Y_{T}=0$ or $\lambda_{T}=1$, the error-and-erasure scheme performs similarly to the error-correction-only decoder, or to the scenario, when no erasure insertion is employed. If the threshold value of $Y_{T}$ is excessive and simultaneously, the threshold value of $\lambda_{T}$ is too low, the system will perform worse than the errorcorrection-only decoding scheme.

The packet forwarding probability versus $E_{b} / N_{0}$ at the relay is seen in Fig. 2. Three decoding schemes were considered, namely the uncoded scheme, error-correction-only decoding and error-anderasure decoding. According to Fig. 2, both of the FEC decoding schemes outperform the uncoded scheme. When error-and-erasure decoding is employed, a packet has a higher chance of being forwarded, compared to the error-correction-only decoder.

Similar to Fig. 1, the codeword decoding error probability recorded at the destination is shown in Fig. 3 at $E_{b} / N_{0}=8 \mathrm{~dB}$. It is also found from the figure that there is an optimum threshold value of $Y_{T}$ or $\lambda_{T}$, which minimizes the decoding error probability.

Fig. 4 compared the achievable performance of the erasure filling and non-erasure-filling decoding schemes for different FSK/ReS schemes. Naturally, the erasure insertion schemes outperform the non-erasure-insertion ones. When employing the $\operatorname{Re} S(31,20)$ code combined with 32-FSK modulation and relying on a single relay, the error-and-erasure decoding scheme achieves a $3 \mathrm{~dB} E_{b} / N_{0}$ gain at $B E R=10^{-4}$, compared to the error-correction-only scheme. Moreover, the ReS code, which employs a high-order Galois field, combined with high-order FSK modulation, perform significantly better above $5 \mathrm{~dB}$, than those using lower-order Galois field combined with low-order FSK modulation.

The system performances of the EGC and SC techniques are compared in Fig. 5. According to the figure, the EGC schemes achieved a lower codeword error probability than the SC arrangement. More particularly, the EGC scheme may achieve a $1.5 \mathrm{~dB}$ gain at $E_{b} / N_{0}$ of $10^{-6}$, compared to the SC scheme, when assisted by a single relay. Likewise, the attainable $E_{b} / N_{0}$ gain is $1.0 \mathrm{~dB}$, when there are two assisting relays. This is not unexpected, because the EGC technique constitutes the optimal diversity combining scheme for non-coherent detection.

Finally, the beneficial effect of the number of relays is shown in Fig. 6. Observe that below $E_{b} / N_{0}$ of $2 \mathrm{~dB}$, the schemes relying on a higher number of relays might in fact perform worse than those relying on a lower number of relays. This is because the total available power is distributed between the source and the relays for the sake of a fair comparison. As a result, the transmit power of both the source as well as the relays may become insufficient for their successful decoding and hence, the packet forward probability at the relay decreased. In other words, the number of relays forwarding data to the destination is reduced. When the $E_{b} / N_{0}$ value increases, more relays forward data to the destination. Thus, the schemes associated with a higher number of relays perform better than those relying on less relays. However, the BER gain associated with employing more than two relays gradually becomes insignificant upon increasing the number of relays. The attainable performance becomes similar to that of the co-located MIMO system upon increasing the number of transmit antennas.

\section{Conclusions}

In this paper, we have proposed a joint MO-RTT erasure insertion aided ReS coded and non-coherently detected SFH MFSK system for cooperative networks. The corresponding mathematical expressions were derived in order to quantify the attainable performance of the proposed system. The results of Fig. 4 showed that the errorand-erasure ReS decoding scheme outperforms the error-correctiononly $\operatorname{ReS}$ decoding arrangement. More particularly, the $\operatorname{Re} S(31,20)$ coded system combined with 32-FSK modulation employing errorand-erasure decoding may achieve an $E_{b} / N_{0}$ gain of approximately $3 \mathrm{~dB}$ at the BER of $10^{-4}$, compared to the same system using error-correction-only decoding. Moreover, we have compared the achievable performance of the system, when employing the EGC and $\mathrm{SC}$ techniques at the receiver. It was found in Fig. 5 that the EGC scheme assisted by one and two relays, is capable of achieving an $E_{b} / N_{0}$ gain of $1.5 \mathrm{~dB}$ and $1.0 \mathrm{~dB}$, respectively, at the BER of $10^{-6}$, compared to the SC scheme.

\section{REFERENCES}

[1] L.-L. Yang and L. Hanzo, "Low complexity erasure insertion in RScoded SFH spread-spectrum communications with partial-band interference and Nakagami-m fading," IEEE Transactions on Communications, vol. 50, pp. $914-925$, June 2002.

[2] S. Ahmed, L.-L. Yang, and L. Hanzo, "Erasure insertion in RS-Coded SFH MFSK subjected to tone jamming and Rayleigh fading," IEEE Transactions on Vehicular Technology, vol. 56, pp. 3563 -3571, Nov. 2007.

[3] A. J. Viterbi, "A robust ratio-threshold technique to mitigate tone and partial band jamming in coded MFSK systems," in IEEE Military Communications Conference - Progress in Spread Spectrum Communications, 1982. MILCOM 1982., vol. 1, pp. 22.4-1 -22.4-5, 17-20 1982.

[4] L. Hanzo, T. H. Liew, and B. L. Yeap, Turbo Coding, Turbo Equalisation and Space-Time Coding for Transmission over Fading Channels. New York, NY, USA: John Wiley \& Sons, Inc., 2002.

[5] L. Hanzo, O. Alamri, M. El-Hajjar, and N. Wu, Near-Capacity MultiFunctional MIMO Systems: Sphere-Packing, Iterative Detection and Cooperation. John Wiley - IEEE Press, May 2009.

[6] R. Steele and L. Hanzo, Mobile Radio Communications: Second and Third-generation Cellular and WATM Systems. John Wiley - IEEE Press, 2nd ed., May 1999.

[7] C. Baum and M. Pursley, "Bayesian methods for erasure insertion in frequency-hop communication systems with partial-band interference," IEEE Transactions on Communications, vol. 40, pp. $1231-1238$, July 1992.

[8] P. Hahn, "Theoretical diversity improvement in multiple frequency shift keying," IRE Transactions on Communications Systems, vol. 10, pp. 177 -184 , june 1962.

[9] J. G. Proakis, Digital communications. McGraw-Hill, 4th ed., 2001.

[10] L.-L. Yang, K. Yen, and L. Hanzo, "A Reed-Solomon coded DS-CDMA system using noncoherent M-ary orthogonal modulation over multipath fading channels," IEEE Journal on Selected Areas in Communications, vol. 18 , pp. $2240-2251$, nov 2000.

[11] I. S. Gradshteyn, A. Jeffrey, and I. M. Ryzhik, Table of integrals, series, and products. New York, NY: Academic Press, 7th ed., 2007. 


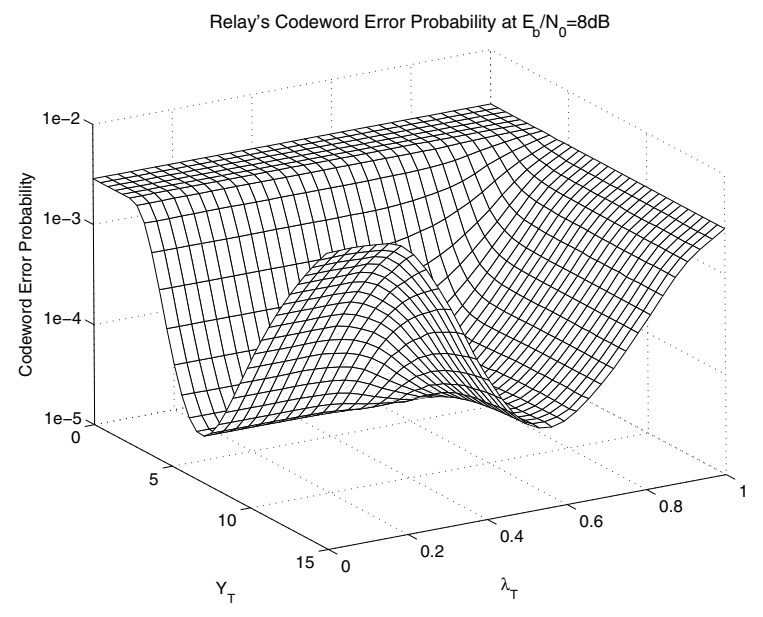

Fig. 1. Codeword decoding error probability at the relay versus the amplitude threshold, $Y_{T}$ and the ratio threshold, $\lambda_{T}$ for the $\operatorname{ReS}(31,20)$ FEC code using "error-and-erasure" decoding based on the MO-RTT erasure insertion scheme for transmission over a flat Rayleigh fading channel: $\rho=0.15, M=$ $32, E_{b} / N_{0}=8 d B, E_{b} / N_{I}=10 d B, L=1, G_{s r}=G_{r d}=4 G_{s d}$.

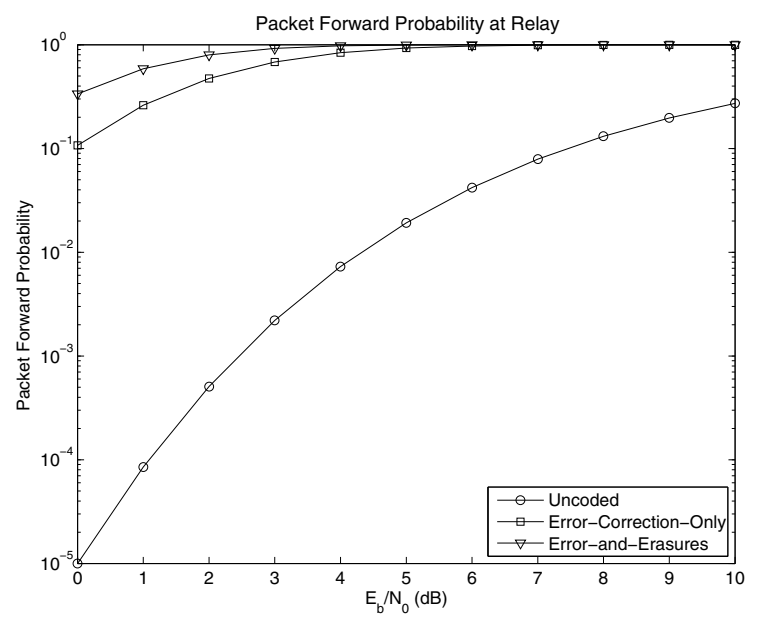

Fig. 2. Packet forward probability at the relay for the $\operatorname{ReS}(31,20)$ FEC code using "error-correction-only" decoding and "error-and-erasure" decoding based on the MO-RTT erasure insertion scheme for transmission over a flat Rayleigh fading channel. The results were evaluated from (6): $\rho=0.15, M=$ $32, E_{b} / N_{I}=10 d B, \operatorname{Re} S(31,20), L=1, G_{s r}=G_{r d}=4 G_{s d}$.

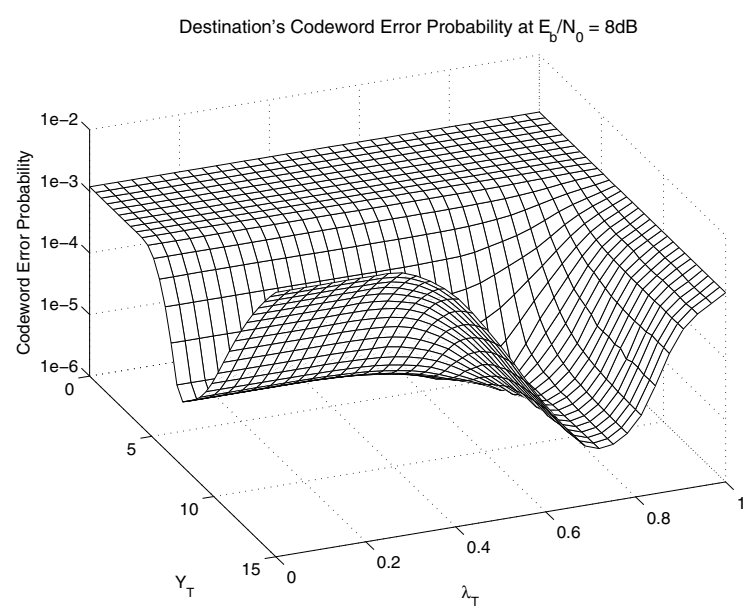

Fig. 3. Codeword decoding error probability at the destination versus the amplitude threshold, $Y_{T}$ and the ratio threshold, $\lambda_{T}$ for the $\operatorname{ReS}(31,20)$ FEC code using "error-and-erasure" decoding based on the MO-RTT erasure insertion scheme for transmission over a flat Rayleigh fading channel. The results were evaluated from (35): $\rho=0.15, M=32, E_{b} / N_{0}=8 d B, E_{b} / N_{I}=$ $10 d B, \operatorname{Re} S(31,20), L=1, G_{s r}=G_{r d}=4 G_{s d}$.

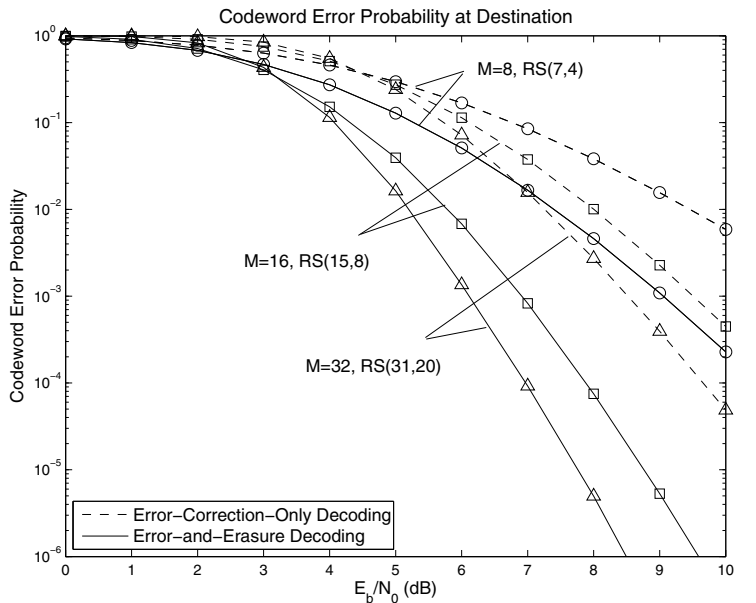

Fig. 4. Codeword decoding error probability at the destination for different ReS FEC codes using "error-correction-only" decoding and "errorand-erasure" decoding based on the MO-RTT erasure insertion scheme for transmission over a flat Rayleigh fading channel. The results were evaluated from (35): $\rho=0.15, E_{b} / N_{I}=10 d B, L=1, G_{s r}=G_{r d}=4 G_{s d}$.

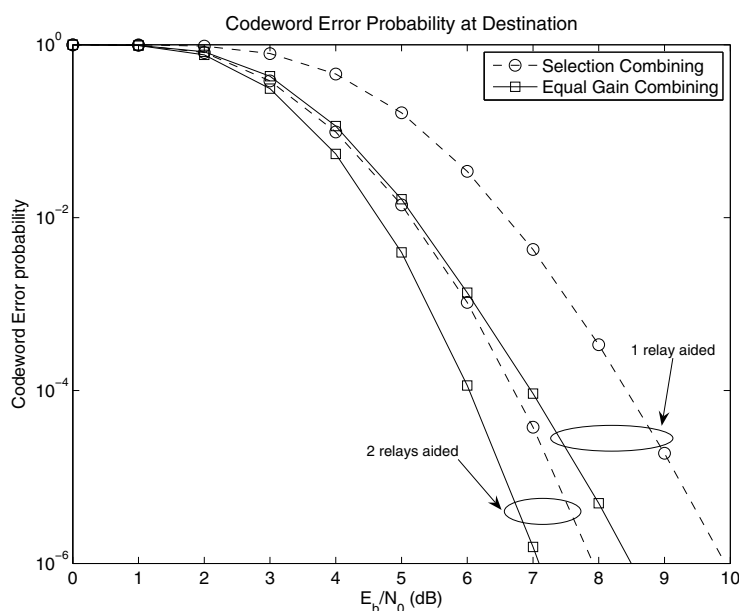

Fig. 5. Codeword decoding error probability at the destination for the $\operatorname{ReS}(31,20)$ FEC codes using "error-and-erasure" decoding based on the MORTT erasure insertion scheme combined with the EGC and SC techniques for transmission over a flat Rayleigh. The results were evaluated from (35): $\rho=0.15, M=32, E_{b} / N_{0}=8 d B, E_{b} / N_{I}=10 d B, \operatorname{Re} S(31,20), L=$ $1, G_{s r}=G_{r d}=4 G_{s d}$.

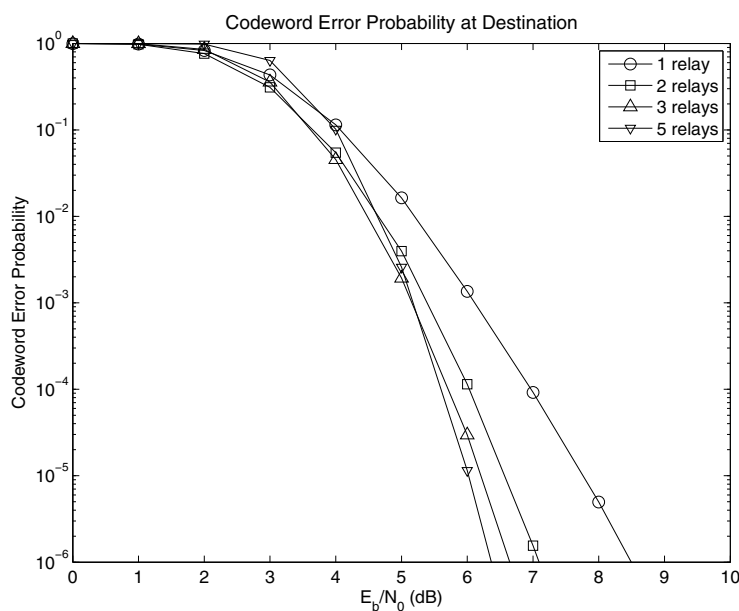

Fig. 6. Codeword decoding error probability at the destination for the $\operatorname{ReS}(31,20)$ FEC codes using "error-and-erasure" decoding based on the MO-RTT erasure insertion scheme for transmission over a flat Rayleigh fading channel when varying the number of relays. The results were evaluated from (35): $\rho=0.15, M=32, E_{b} / N_{0}=8 d B, E_{b} / N_{I}=$ $10 d B, \operatorname{Re} S(31,20), L=\{1,2,3,5\}, G_{s r}=G_{r d}=4 G_{s d}$. 